\section{Proarrhythmia assessment in treatment with hydroxychloroquine and azithromycin hospitalized elderly COVID-19 patients - our experience} Carlo Torchia, ${ }^{1}$ Carmen Ruberto, ${ }^{1}$ Viviana Vespertini, ${ }^{3}$ Lucio Cosco, ${ }^{3}$ Giovanni Ruotolo ${ }^{1}$

${ }^{1}$ Geriatric Unit, 'Pugliese-Ciaccio' General Hospital, Catanzaro; ${ }^{2}$ Primary Care Department, Azienda Sanitaria Provinciale di Catanzaro; ${ }^{3}$ Infectious Diseases Unit, 'Pugliese-Ciaccio' General Hospital, Catanzaro, Italy

\section{Abstract}

The aim of our study was to characterize the repolarization disorders propensity induced by drug-drug interaction. In this observational retrospective study, we report our experience on all elderly patients with ascertained diagnosis of coronavirus disease 2019 through nasopharyngeal swab with real time-polymerase chain reaction at our Pugliese-Ciaccio hospital in Catanzaro, who received hydroxychloroquine (HCQ), with or without azithromycin (AZY). 33 hospitalized patients were examined. We calculated QT value, cQT, QT dispersion, and cQT dispersion and examined possible progression on the basal electrocardiogram (T0) and after the insertion of the drug (T1). The QT value is increased by T0 vs T1 $(370 \pm 40.74$ vs $420 \pm 36.91 \mathrm{~ms} ; \mathrm{P}=0.000$ ), as well as the cQT value ( $408 \pm 25.40$ vs 451.54 \pm 58.81 ; $\mathrm{P}=0.003$ ), the $\mathrm{QT}$ dispersion (QTd: $36.36 \pm 14.53$ vs $50.90 \pm 13.12 \mathrm{~ms} ; \mathrm{P}=0.000$ ); the dispersion of cQTc (cQTd 46.27 \pm 18.72 vs $63.18 \pm 21.93 \mathrm{~ms} ; \mathrm{P}=0.001)$. The $\Delta \mathrm{QT}$ was $37.44 \pm 44.09$ while the $\Delta \mathrm{cQT}$ was $32.01 \pm 56.47)$. The main determinant of QTc prolongation is the number of drug at risk of prolongation of the QT that could influence the ventricular repolarization phase. The use of HCQ in combination with AZY, in patients suffering from severe acute respiratory syndrome-related coronavirus- 2 , can favor the onset of serious side effects, even potentially fatal. Finally, the measures of QTd and cQTd confirmed additional electrocardiographic parameters useful in identifying patients being treated with drugs at risk of potential adverse arrhythmic events following drug interaction.

\section{Introduction}

As of 17 October 2020, the number of severe acute respiratory syndrome-related coronavirus-2 (SARS-CoV-2) infected subjects in the world is 39.5 million, with a mortality of 1.11 million patients (3.09\%). As we still do not have an effective therapy for SARS-CoV-2 patients are treated with several non-specific drugs. Preliminary studies had shown a promising effect of high-dose hydroxychloroquine (HCQ) in reducing SARS-CoV-2 viral load in hospitalized coronavirus disease 2019 (COVID19) patients, with enhanced effects in combination with azithromycin (AZY) or various antiviral drugs. ${ }^{1,2}$ HCQ is an older antimalarial drug, first approved in 1955 by the United States Food and Drug Administration (FDA), also used in longterm treatment of connective tissue diseases, widely available and economically viable and has been regarded as generally safe and well tolerated in patients treated for chronic inflammation. It is postulated to exert a direct antiviral activity by increasing intracellular $\mathrm{pH}$ resulting in decreased phago-lysosome fusion, impairing viral receptor glycosylation. In addition, it has immune-modulating effect by inhibiting toll-like receptor signaling, decreasing production of cytokines especially interleukin (IL)- 1 and IL-6. ${ }^{3}$ Prior data also suggests a potential anti-thrombotic effect. ${ }^{4}$ However, HCQ prolongs the QT interval due to blocking of the potassium internal cell current. ${ }^{5-7}$ This effect may be particularly enhanced when combined with other drugs which may in themselves prolong the QT interval, with a consequent increased risk of druginduced proarrhythmia, conduction block and ventricular arrhythmias, sometimes fatal. Azithromycin, a macrolide antibiotic has in vitro antiviral properties such as decreased viral replication, blocking entrance into host cells, and a potential immunomodulating effect. ${ }^{8}$ An in vitro study demonstrated synergistic activity of the combination of hydroxychloroquine and azithromycin against SARS-CoV-2. ${ }^{9}$ Although widely used, azithromycin has also been increasingly recognized for risks of QT interval prolongation and sudden death. The information relating to the safety of the use of these drugs, alone or in combination in elderly COVID-19 patients, particularly if in polypharmacy, in addition to being contradictory, indicated an uncertain benefit in terms of efficacy with a safety profile burdened by risks. The US FDA as of June 15, 2020 has revoked the prior emergency use authorization to use hydroxychloroquine and chloroquine to treat
Correspondence: Alberto Castagna, Primary

Care Department, Azienda Sanitaria

Provinciale di Catanzaro, viale Crotone, 88100 Catanzaro, Italy.

Tel./Fax: +39.096.17033013

E-mail: albertocastagna@tiscali.it

Key words: QT assessment; hydroxychloroquine; azithromycin; COVID-19.

Contributions: RC, AC, CT, CR, VV, LC GR, literature research; RC, AC, GR, manuscript writing, conception and critical revisions.

Conflict of interests: the authors declare no potential conflicts.

Availability of data and materials: the data supporting the findings of this study are available from the corresponding author upon reasonable request.

Ethics approval and consent to participate: the local Ethic Committee approved the study and all patients gave written informed consent.

Received for publication: 28 November 2020 . Revision received: 12 February 2021.

Accepted for publication: 9 March 2021.

This work is licensed under a Creative Commons Attribution-NonCommercial 4.0 International License (CC BY-NC 4.0).

${ }^{\circ}$ Copyright: the Author(s), 2021

Licensee PAGEPress, Italy

Geriatric Care 2021; 7:9536

doi:10.4081/gc.2021.9536

COVID-19 in certain hospitalized patients when clinical trial data is unavailable or participation is not feasible..$^{10}$ Currently, in light of the latest results available in literature, the authorization to use the HCQ offlabel, outside of clinical studies, has been suspended.

\section{Materials and Methods}

In this observational retrospective study, we report our experience on all elderly patients with ascertained diagnosis of COVID-19 through nasopharyngeal swab with real time-polymerase chain reaction (RT-PCR), who arrived from 1 March 2020 to 30 June 2020 at our Pugliese-Ciaccio Hospital in Catanzaro, who received HCQ, with or without AZY, in the absence of contraindications and prescribing limitations in force at that time, including an excessive prolongation of the baseline $\mathrm{QT} / \mathrm{cQT}>450 \mathrm{~ms}$, and which they assumed, due to their comorbidities (diabetes mellitus, arterial hypertension, and 
dementia with behavioral disorders), several drugs some of which are at risk of causing this side effect. Each patient, after entering the ward, was periodically monitored also through electrocardiographic traces who were manually evaluated by cardiologists to calculate cQT intervals using the Bazett's formula.

\section{The aim of our study}

was to characterize the repolarization disorders propensity induced by drug-drug interaction.. We also evaluated the change in QT and cQT from baseline $(\Delta \mathrm{QT}$ and $\Delta \mathrm{cQT}$ respectively) and QT and cQT dispersion (QTd and cQTd), calculated as the difference between the value of QT and maximum cQT and the value of the minimum QT and cQT, respectively, recorded in the twelve leads of an ECG. The verification was carried out by comparing the ECG trace in the baseline at the entrance, (T0) and after the use of HCQ (T1), alone or in association with AZY. The dose of HCQ was $400 \mathrm{mg}$ twice daily for 2 doses on day 1, followed by $200 \mathrm{mg}$ twice daily on days 2-5. Azithromycin was dosed as $500 \mathrm{mg}$ once daily on day 1 followed by $250 \mathrm{mg}$ once daily for the next 4 days. For patients with more severe forms of COVID-19 and with minimal cardiovascular risk factors, the combination HCQ + AZY was reserved. cQT $>500 \mathrm{~ms}$ was considered an elevated cardiac risk

\section{Results}

We retrospectively analyzed ECG traces of 33 elderly hospitalized SARSCoV-2 patients (RT-PCR), before and after HCQ binding; AZY was associated with 16 patients $(73 \%)$. The general descriptive characteristics of the study population are shown in Table 1. The QT value is increased by T0 vs T1 $(370 \pm 40.74$ vs $420 \pm 36.91 \mathrm{~ms}$; $\mathrm{P}=0,000)$, as well as the cQT value (408 \pm 25.40 vs $451.54 \pm 58.81 ; \mathrm{P}=0.003)$, the QT dispersion (QTd: $36.3+14.6$ msec $v s 51$ $+13.4 \mathrm{msec} \mathrm{P}=0.000)$; the dispersion of cQTc (cQTd 46.27 \pm 18.72 vs $63.18 \pm 21.93$ $\mathrm{ms} ; \mathrm{P}=0.001)$. The $\Delta \mathrm{QT}$ was $37.44 \pm 47.09$ while the $\Delta \mathrm{cQT}$ was $32.01 \pm 56.47$ ) (Table 2). Among patients taking at least two drugs with potential risk of QTc prolongation, excessive prolongation of cQT $>500 \mathrm{msec}$ and an increase in $\Delta \mathrm{cQT}>60 \mathrm{msec}$ in 4 patients $(16.66 \%)$ was recorded from $\mathrm{T} 0$ to $\mathrm{T} 1$, thus as an increase in QT and cQT interval dispersion (QTd and cQTd) in 13 patients $(54.16 \%)$. The univariate ANOVA analysis between the two HCQ vs HCQ + AZY groups did not show significant differ- ences. We have also created multiple regression models with the main electrocardiographic parameters as a dependent variable, inserting demographic parameters, multidimensional assessment parameters, the number of drugs taken and the number of drugs at risk of prolongation of the QT interval in the model. It is important to underline how the model, with the dependent variable delta cQT, has identified $>2$ QTc prolonging drugs (beta $0.711 ; \mathrm{P}=0.002$ ) as a directly statistically correlated variable (Table 3 ). Although this model shows that the main determinant of QTc prolongation is the

number of drug at risk of prolongation of the QT that could influence the ventricular repolarization phase.

\section{Discussion}

The choice of care for patients with COVID-19, especially in the most severe forms and in the elderly, still places medical teams in front of complex decisions. The difficulties are greater in consideration of the lack of proven therapeutic strategies.

Table 1. Demographic, clinical and comprehensive geriatric assessment data in hospitalized older patients with COVID-19.

\begin{tabular}{lc}
\hline Age, Years & $75.97 \pm 8.48$ \\
Sex, M\% & $30.3 \%$ \\
\hline Presenting symptoms & $75.8 \%$ \\
Fever,\% & $97 \%$ \\
Dyspnea,\% & $30.3 \%$ \\
GI symptoms,\% & $69.7 \%$ \\
Cough,\% & $4.87 \pm 3.07$ \\
SPMSQ & $2.18 \pm 2.28$ \\
\hline ADL & $2.06 \pm 3.11$ \\
IADL & $5.39 \pm 2.70$ \\
CIRS & $12.69 \pm 3.25$ \\
ESS & $8.53 \pm 2.70$ \\
\hline MNA & $7.84 \pm 2.41$ \\
Drugs, n & $5.39 \pm 2.70$ \\
CIRS & $87.87 \%$ \\
Hypertension, $\%$ & $15.15 \%$ \\
Diabetes;\% & $24.24 \%$ \\
Dyslipidemia, $\%$ & $21.21 \%$ \\
COPD, \% & $12.12 \%$ \\
Heart failure, \% & $18.18 \%$ \\
Previous MI, \% &
\end{tabular}

GI, gastrointestinal; SPMSQ, short portable mental status questionnaire; ADL, activities of daily living; IADL, instrumental activities of daily living; ESS, Exton-Smith scale; MNA-SF, mini nutritional assessment-short form; CIRS, comorbidity index rating scale; COPD, chronic obstructive pulmonary disease; MI, myocardial infarction.

Table 2. Electrocardiogram: QT analysis in T0 and T1 in hospitalized older patients with COVID-19.

\begin{tabular}{lccc} 
& T0 & T1 & P-value \\
QT, ms & $370.09 \pm 40.74$ & $407.54 \pm 36.91$ & 0.000 \\
cQT, ms & $419.54 \pm 25.40$ & $451.54 \pm 59.28$ & 0.003 \\
\hline QT Dispersion, ms & $36.36 \pm 14.53$ & $50.90 \pm 13.41$ & 0.0000 \\
cQT Dispersion, ms & $46.27 \pm 18.72$ & $63.18 \pm 23.11$ & 0.0001 \\
\hline$\Delta$ QT, ms & & $37.44 \pm 47.09$ & \\
$\Delta$ cQT, $m s$ & $32.00 \pm 56.47$ & \\
\hline
\end{tabular}

Table 3. Multivariate stepwise logistic regression analysis with $\Delta$ QTc as dependent variable, in hospitalized older patients with COVID-19.

\begin{tabular}{lcc} 
& Beta & P-value \\
$>2$ QTc prolonging drugs & 0.711 & 0.002 \\
\hline
\end{tabular}


Among the possible therapies, hydroxychloroquine, thanks to its anti-inflammatory and antiviral properties, had shown promise in fighting the infection, but also burdened by non-negligible safety problems, especially if used in combination with other drugs at risk of QT prolongation such as AZY. It is known as, in geriatric care, the main concern about the use of HCQ are QTC prolongation and the addictive effect when used with the drugs largely used in older patients. Moreover, the large majority of trials with HCQ have been conducted in Europe or USA in young people which did not show relevant comorbidities and drug interactions. ${ }^{11}$ In some studies, drug combinations that prolong the cQT interval did not result in a greater degree of prolongation of the cQT interval than single drug administration. ${ }^{12}$ It is not uncommon for patients with a prolonged cQT interval to be routinely prescribed drugs that prolong the cQT interval, despite the increased risk. ${ }^{13,14}$ Our data confirmed the prolongation of the cQT interval following the use of HCQ, and that the magnitude of the increase in cQT and $\triangle \mathrm{cQT}$ from baseline was more pronounced in patients treated with HCQ especially in combination with additional drugs potentially at risk of prolonging the cQT interval, including AZY. The repolarization disturbance, detected with the different parameters derived from the ECG trace, could have been further amplified in the context of COVID-19 as well as due to the drug-drug interaction (HCQ, AZY, antivirals, antipsychotics), also by the clinical characteristics acute infection which can manifest itself with fever, dehydration and electrolyte abnormalities. Furthermore, SARS-CoV-2 is responsible for an acute respiratory infection which, through the triggered cytokine storm, could present with acute cardiovascular events such as myocarditis or acute coronary syndrome, further amplifying cardiovascular and systemic risks. ${ }^{15,16}$ The patients in our study, elderly with COVID-19, were seriously ill, some of them even with severe personality disorders, for which they were exposed to multiple drugs with prolonged half-lives and at risk of cardiotoxicity detectable also through the prolongation of the cQT interval. In any case, none of our patients experienced severe arrhythmias or sudden death. Our data are in agreement with those present in recent studies that have appeared on the subject. The Reports from Lyon, France and Boston, Massachusetts, provide concordant insights into the cQT interval prolongation potential with this treatment in patients admitted with COVID-19, where they demonstrated a high percentage of cQT prolongation of patients treated with HCQ especially when in combination with AZY. ${ }^{17,18}$ The prolongation of the QT interval, of the cQT detected on the ECG, is known to reflect the prolongation of ventricular repolarization and therefore the effective refractory period and commonly occurs in patients in cardiac care units. Many factors influence the duration of ventricular repolarization, and the QT interval varies from beat to beat, day to day and day to night. It is influenced by several factors such as age, sex, electrolyte concentrations, autonomic tone, myocardial ischemia and especially by different classes of drugs. The dispersion of QT and cQT (QTd and cQTd), which we have included in our measurements, although it is currently recognized as an indirect index of gross anomalies of ventricular repolarization, at the same time its calculation undoubtedly appears useful in different pathological contexts and in their delicate therapeutic management, sometimes representing an additional value of further aid in order to prevent the possibility of developing iatrogenic cardiac toxicity. ${ }^{19}$ Furthermore, since the univariate ANOVA analysis between the two groups HCQ vs HCQ + AZY was not significant, in our population under examination it was not the association of AZY to HCQ that influenced the prolongation of the QT interval, cQT and increased dispersion of the QT interval. Although TdP occurs relatively rarely, it is a catastrophic event in hospitalized patients and, due to the severity of this proarrhythmia, the AHA/ACCF strongly recommends increased focus on QT interval prolongation, TdP risk and QT interval monitoring and QT avoidance drugs that prolong the interval wherever possible in hospitalized patients. ${ }^{20}$ Moreover, It was also shown that, in a group of COVID-19 subjects requiring oxygen therapy, on day 21 of HCQ therapy, there was no difference, compared to the control, in the survival rate in patients who were not transferred to ICU, in their overall survival, survival without acute respiratory distress syndrome and weaning from oxygen. ${ }^{21}$ In an observational study involving hospitalized patients with COVID-19, HCQ administration was not associated with either a greatly lowered or an increased risk of the composite end point of intubation or death. ${ }^{22}$

The evidence currently available regarding the dubious efficacy of HCQ and AZY in the treatment and prophylaxis of SARS-CoV-2 infection, and that relating to the safety profile, burdened by potential risks, have required the suspension of HCQ alone or in association with other drugs outside of clinical trials.

\section{Conclusions}

This study, which evaluated data obtained during real life in elderly subjects affected by COVID-19, albeit in the presence of a low number of subjects, highlighted the difficulties in managing these patients, who often carry the burden of multiple morbidity with them. and polypharmacy, placing us in front of complex choices. The data also confirmed that the use of HCQ in combination with AZY, in patients suffering from SARS-CoV-2, can favor the onset of serious side effects, even potentially fatal. Finally, the measures of QTd and cQTd confirmed additional electrocardiographic parameters useful in identifying patients being treated with drugs at risk of potential adverse arrhythmic events following drug interaction. Overall, the results of clinical trials seem to demonstrate a substantial absence of clinical benefit of HCQ both in the hospitalized population with moderate-severe COVID-19 disease and in the less advanced one, in the face of an increase in adverse events, albeit not serious. These considerations have limited the use of the drug in hospitalized patients due to SARS-CoV-2 infection.

\section{References}

1. Gautret P, Lagier JC, Parola P, et al. Clinical and microbiological effect of a combination of hydroxychloroquine and azithromycin in 80 COVID-19 patients with at least a six-day followup: one pilot observational study. Travel Med Infect Dis. 2020:101663. [Epub ahead of print].

2. Castagna A, Vetta F, Attisani G, et al. Hydroxychloroquine and QTc: beyond COVID-19; Geriatric Care 2020;6:9064.

3. Savarino A, Boelaert JR, Cassone A, et al. Effects of chloroquine on viral infections: an old drug against todays diseases. Lancet Infect Dis 2003;3:722-7.

4. Jung H, Bobba R, Su J, et al. The protective effect of antimalarial drugs on thrombo-vascular events in systemic lupus erythematosus. Arthrit Rheum 2010;62:863-8.

5. Chen CY,Wang FL, Lin CC. Chronic hydroxychloroquine use associated with QT prolongation and refractory ventricular arrhythmia. Clin Toxicol (Phila). 2006;44:173-5.

6. O'Laughlin JP, Mehta PH,Wong BC. Life threatening severe QTc prolongation in patient with systemic lupus erythematosus due to hydroxychloroquine. 
Case Rep Cardiol 2016;2016:4626279.

7. World Health Organization. The cardiotoxicity of antimalarials. Geneva: World Health Organization Malaria Policy Advisory Committee Meeting; March 22, 2017. Available from: https://www.who.int/malaria/mpac/mpa c-mar2017-erg-cardiotoxicity-reportsession2.pdf Accessed: 22 April, 2020.

8. Tran DH, Sugamata R, Hirose T, et al. Azithromycin, a 15-membered macrolide antibiotic, inhibits influenza $\mathrm{A}(\mathrm{H} 1 \mathrm{~N} 1) \mathrm{pdm} 09$ virus infection by interfering with virus internalization process. J Antibiot 2019;72:759-68.

9. Andreani J, Bideau ML, Duflot I, et al. In vitro testing of combined hydroxychloroquine and azithromycin on SARS-CoV-2 shows synergistic effect. Microb Pathog 2020;145:104228.

10. Food and Drug Administration (FDA). FDA cautions against use of hydroxychloroquine or chloroquine for COVID19 outside of the hospital setting or a clinical trial due to risk of heart rhythm problems; June 15, 2020 Update. Available from: https://www.fda.gov/ drugs/drug-safety-and-availability/fdacautions-against-use-hydroxychloroquine-or-chloroquine-covid-19-outsidehospital-setting-or

11. Cenderello G, Dini S, Ziliani S, et al. COVID-19 treatment in the older people: a brief clinical review. Geriatric Care 2020;6:9330.

12. Charbit B, Alvarez JC, Dasque E, et al.
Droperidol and ondansetron-induced QT interval prolongation: a clinical drug interaction study. Anesthesiology 2008;109:206-12.

13. Tisdale JE, Wroblewski HA, Overholser BR, et al. Prevalence of QT interval prolongation in patients admitted to cardiac care units and frequency of subsequent administration of QT interval-prolonging drugs. A prospective, observational study in a large urban academic medical center in the US. Drug Saf 2012;35:459-70.

14. Costa R, Castagna A, Torchia C, et al. COVID-19 and cardiovascular disease in elederly patients: a challenge in the challenge. Geriatric Care 2020;6:9121.

15. Warren-Gash C, Blackburn R, Whitaker $\mathrm{H}$, et al. Laboratory-confirmed respiratory infections as triggers for acute myocardial infarction and stroke: a selfcontrolled case series analysis of national linked. Eur Respir J 2018;51:1701794.

16. Bodini G, Demarzo MG, Casagrande E, et al. Concerns related to COVID-19 pandemic among patients with inflammatory bowel disease, and its influence on patients management. Eur J Clin Invest 2020; 13233. [Epub ahead of print].

17. Bessière $F$, Roccia $H$, Delinière $A$, et al. Assessment of QT Intervals in a case series of patients with coronavirus disease 2019 (COVID-19) infection treated with hydroxychloroquine alone or in combination with azithromycin in an intensive care unit. JAMA Cardiol 2020:1787. [Epub ahead of print].

18. Mercuro NJ, Yen CF, Shim DJ, et al. Risk of QT interval prolongation associated with use of hydroxychloroquine with or without concomitant azithromycin among hospitalized patients testing positive for coronavirus 2019 (COVID-19) infection. JAMA Cardiol 2020:1834. [Epub ahead of print].

19. Malik M, Batchvarov VN. Measurement, interpretation and clinical potential of QT dispersion. J Am Coll Cardiol 2000;36:6.

20. Drew BJ, Ackerman MJ, Funk M, et al.; on behalf of the American heart Association Acute Cardiac care Committee of the Council on Clinical Cardiology, the Council on Cardiovascular Nursing, and the American College of Cardiology Foundation. Prevention of torsade de pointes in hospital settings: a scientific statement from the American Heart Association and the American College of Cardiology Foundation. J Am Coll Cardiol 2010;55:934-47.

21. Mahévas M, Tran VT, Roumier M, et al. Clinical efficacy of hydroxychloroquine in patients with COVID-19 pneumonia who require oxygen: observational comparative study using routine care data. BMJ 2020;369:m1844.

22. Geleris J, Sun Y, Platt J, et al. Observational study of hydroxychloroquine in hospitalized patients with covid19. N Engl J Med 2020;382:2411-8. 